\title{
Some aspects of clonal micropropagation of Amelanchier Medik. genus representatives
}

\author{
Ekaterina N. Raeva-Bogoslovskaya ${ }^{1,{ }^{*}}$, Olga I. Molkanova ${ }^{1}$, and Viktoriya A. Kryuchkova ${ }^{2}$ \\ ${ }^{1}$ FSBIS Main Botanical Garden named after N.V. Tsitsin, RAS, Laboratory of Plant Biotechnology, \\ 127276, Moscow, Russia \\ ${ }^{2}$ FSBIS Main Botanical Garden named after N.V. Tsitsin, RAS, Laboratory of Cultured Plants, \\ 127276, Moscow, Russia
}

\begin{abstract}
The article presents studies of organogenesis peculiarities of varieties belonging to various species of Amelanchier Medik. genus in in vitro culture at the stages of micropropagation, rooting and depositing. Mineral composition and growth regulator for active adventive microshoots formation were determined. The optimal auxin source for rhizogenesis of various Amelanchier alnifolia varieties has been established. Conditions for explants' preservation in aseptic conditions for 12 months have been revealed. Different effects of cytokinin type substances on shadberry regeneration potential under aseptic conditions were noted. Analysis of regenerants' morphometric indicators showed the effectiveness of using 6-benzylaminopurin (BAP) in the concentration of $1.0 \mathrm{mg} / \mathrm{L}$ for shadberry varieties' clonal micropropagation and indolebutyric acid (IBA) at $1.0 \mathrm{mg} / \mathrm{L}$ concentration for microshoots' rooting.
\end{abstract}

\section{Introduction}

Every year, the need for high-yield fruit and berry plantations resistant to various environmental factors is constantly increasing. However, in order to improve the quality of life, the diversity of the product range is also important. The solution of such problems requires the introduction of new species of plants with economically valuable properties. For Eurasia, one such perspective crops is shadberry (Amelanchier Medik.), which grows mainly in the territory of North America. Species of this genus are shrubs or small trees with edible dark blue fruits ripening in late June-August. Shadberry raw materials is characterized by a high content of biologically active substances and have been widely used in various industries in the United States and Canada.

It was found that in comparison with bird-cherry, chokeberry and rowan, shadberry fruits are characterized by the highest content of sugars $(10.0-13.5 \%)$. It should be noted that representatives of Amelanchier genus also exceed raspberries, blueberries and

\footnotetext{
${ }^{*}$ Corresponding author: katyaraeva@rambler.ru
} 
honeysuckle by this indicator [1]. Consequently, there is a possibility of using residual shadberry processing products as a substitute for sugar in flour products [2]. Due to the high anthocyanin content in fruits, shadberry is used as a natural food dye $[3,4]$. In addition to the rich biochemical composition, the fruits have a harmonious taste. Juices, wines, compotes, jams, and marmalade are prepared from them $[5,6]$.

Also, shadberry is used for medicinal purposes. Fruits can be used to produce natural multivitamin concentrates and therapeutic and preventive purposes products [7]. Some researchers noted high flavonols content in shadberry fruits in comparison with other berry crops [8]. Antioxidant properties of alcohol extracts of various plant parts were revealed. [9].

Shadberry reproduction is carried out by seeds, root offspring, bush division, cuttings, grafting and biotechnology methods $[3,5,10,11]$. Seed reproduction is suitable for obtaining planting material of only plant species. Root offspring and bush division allow to get no more than 6 daughter plants [12]. This makes these methods ineffective for breeding shadberry varieties. Such methods as grafting and cuttings heavily depend on the time of year and also require the presence of rootstock plantations $[5,6]$. One of the methods of accelerated equated planting material obtaining is clonal micropropagation [11].

Some stages of clonal micropropagation have been previously studied by scientists on a small group of shadberry varieties and species. Thus, micropropagation of four varieties of A. alnifolia species revealed the application effectiveness of the nutrient medium Murashige-Skoog (MS) in comparison with Gamborg (1968), Nitsch (1968) and advantage of BAP over other sources of cytokinin (thidiazuron, zeatin and kinetin) [13, 14]. Such physiological disturbance as vitrification was studied on the example of $A$. arborea. It was found that the vitrification degree decreased with higher agar concentration and increased when ammonium nitrate was added [15]. The influence of various sources of iron and auxin action-type substances on the growth, development and rooting of A. alnifolia in in vitro culture has been determined. The MS nutrient medium containing FeNaEDTA significantly increased the number of microshoots [16]. At the same time, the largest percentage of rooted microshoots was obtained on the $1 / 2$ MS nutrient medium with the addition of 1.0 $\mathrm{mg} / \mathrm{L}$ naphthylacetic acid [17].

To date, organogenesis of Amelanchier genus representatives has not been fully studied and the in vitro cultivation technique requires improvement, which makes the research in this direction relevant.

The purpose of the scientific work was to identify morphogenesis features and compare the regeneration potential of Amelanchier genus representatives at the stages of micropropagation and rooting depending on used mineral compositions of nutrient media and growth regulators.

\section{Objects and methods of research}

In the in vitro plants' collection of the plant biotechnology laboratory of the FSBIS MBG named after N. V. Tsitsin RAS, varieties belonging to various species of Amelanchier Medik.: 'Krasnoyarskaya', 'Mandan' (A. alnifolia (Nutt.) Nutt. genus are being bred and preserved. Ex M.Roem.); × Amelasorbus Rehder; 'Prince William' (A. canadensis (L.) Medik.); 'Ballerina' (A. × grandiflora Rehder).

Quorin and Lepoivre (QL) (1977), Woody Plant Medium (WP) (1980) and MurashigeSkoog (MS) (1962) were tested to determine the optimal mineral composition of the nutrient medium, the latter was used as control. BAP was added to each nutrient medium option at a concentration of $0.5 \mathrm{mg} / \mathrm{L}$.

To identify development peculiarities of shadberry explants depending on the cytokinin source, two growth regulators were compared: 6-benzylaminopurin (BAP) and 2-isopentyl- 
adenine (2-IP) at $1.0 \mathrm{mg} / \mathrm{L}$ concentrations. As a control, some of the explants were placed on MS's hormone-free $(\mathrm{H} / \mathrm{f})$ nutrient medium. Further, BAP was tested at a concentration of $0.2 ; 0.3 ; 0.5 ; 1.0 \mathrm{mg} / \mathrm{L}$. A control option of MS with the addition of $0.5 \mathrm{mg} / \mathrm{L} \mathrm{BAP}$.

During the rooting phase, the explants were transferred to a nutrient medium containing $1 / 2$ of the MS mineral composition, as well as indolebutyric (IBA) and indoleacetic (IAA) acids at concentrations of 0.5 and $1.0 \mathrm{mg} / \mathrm{L}$. Control option without growth regulator. During cultivation, the following conditions were maintained: lighting $2000 \mathrm{~lx}$, photoperiod $16 / 8$ hours, temperature $23-25^{\circ} \mathrm{C}$.

After 28 days at micropropagation stage, microshoots height measurement, visual counting of their number and multiplication factor calculation were performed; the latter is the sum of explants' number obtained during the division of newly formed microshoots. The microshoots' height and their number allow to predict the rooting stage, and the multiplication factor - the stage of micropropagation itself. At the rhizogenesis stage, the roots formed in regenerant plants were counted and the percentage of rooting was calculated.

Generally accepted methods of statistical analysis implemented using Microsoft Office Excel 2010 and PAST 2.17 software were used to process the obtained data.

\section{Results and Discussion}

Organogenesis in in vitro culture is known to be controlled by many factors. The study of the genotype effect on the number of microshoots and their height (Table 1) was conducted in the experiment.

Table 1. Genotype effect on morphometric indicators of shadberry explants

\begin{tabular}{|c|c|c|}
\hline \multirow{2}{*}{ Morphometric indicators } & \multicolumn{2}{|c|}{ Genotypes } \\
\cline { 2 - 3 } & 'Krasnoyarskaya' & 'Mandan' \\
\hline $\begin{array}{c}\text { Number of microshoots, pcs. } \\
\text { LCD } 050.6\end{array}$ & 2.5 & 21.8 \\
\hline $\begin{array}{c}\text { Height of microshoots, mm. } \\
\text { LCD } 053,4\end{array}$ & 13.3 & 21.7 \\
\hline
\end{tabular}

During the two-factor analysis, the varietal specificity was established for such indicators as the number and height of microshoots in A. alnifolia. varieties. The mineral composition of the nutrient medium did not have a significant impact on these traits. 'Krasnoyarskaya' variety exceeds 'Mandan' variety by the number of microshoots $(2.5 \mathrm{pcs}$ and 1.8 pcs. respectively), but is inferior in microshoots' height $(13.3 \mathrm{~mm}$ and $21.7 \mathrm{~mm})$. The mineral basis of the nutrient medium had a significant impact on the multiplication factor (Fig.1). 


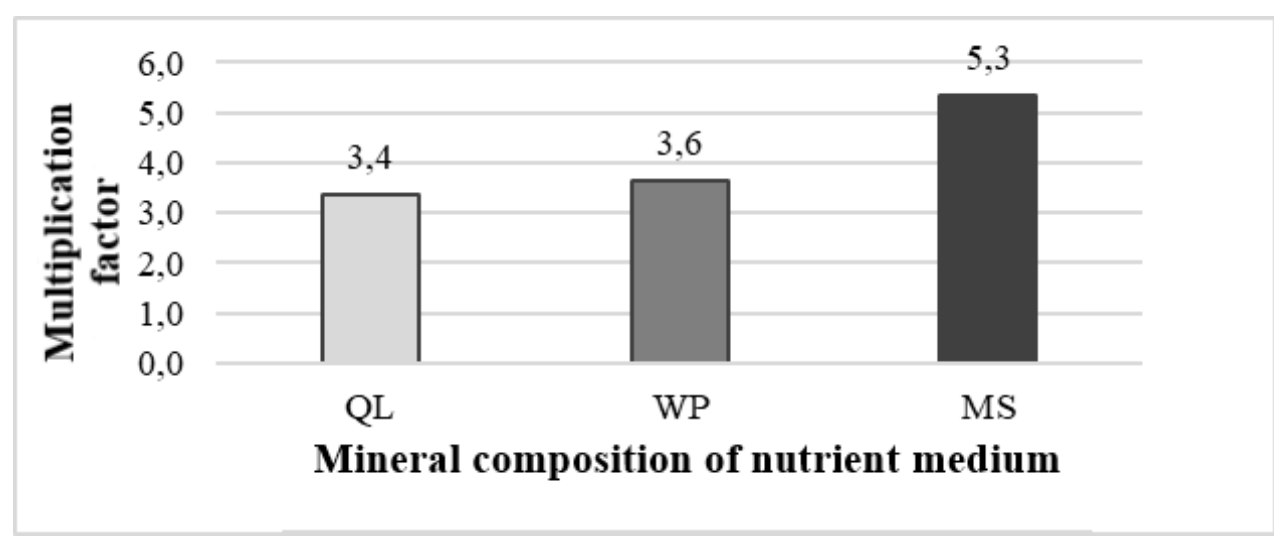

Fig. 1. Influence of the nutrient medium mineral basis on the multiplication factor A. alnifolia, $\mathrm{LCD}_{05} 1.42$

The multiplication factor achieved on the MS control nutrient medium (5.3) was 1.5 times the multiplication factor at QL (3.4) and WP (3.6). These results are consistent with the research of other authors [13]. Thus, MS nutrient medium was used for subsequent experiments.

Maintaining an actively proliferating culture largely depends on the correct selection of cytokinin source. During the comparative analysis, the significant influence of the growth regulator on the regeneration potential of shadberry varieties (Table 2) was revealed.

Table 2. Influence of growth regulator on morphometric indicators of shadberry explants

\begin{tabular}{|c|c|c|c|c|c|c|c|c|c|}
\hline \multirow{2}{*}{ Genotypes } & \multicolumn{3}{|c|}{$\begin{array}{c}\text { Number of } \\
\text { microshoots, pcs. } \\
\text { LCD } 05 \text { 0,4 }\end{array}$} & \multicolumn{3}{c|}{$\begin{array}{c}\text { Height of } \\
\text { microshoots, mm. } \\
\text { LCD } 051,8\end{array}$} & \multicolumn{4}{c|}{$\begin{array}{c}\text { Multiplication factor } \\
\text { LCD } 050.4\end{array}$} \\
\cline { 2 - 12 } & BAP & $2-I P$ & H/f & BAP & 2 -IP & H/f & BAP & 2 -IP & H/f \\
\hline 'Krasnoyarskaya' & 4.8 & 1.0 & 1.0 & 14.5 & 12.7 & 9.6 & 7.1 & 1.2 & 1.0 \\
\hline 'Mandan' & 3.4 & 1.0 & 1.0 & 13.5 & 11.3 & 9.5 & 4.3 & 1.3 & 1.0 \\
\hline 'Prince William' & 1.9 & 1.0 & 1.0 & 18.6 & 14.1 & 12.1 & 2.4 & 1.2 & 1.0 \\
\hline Amelasorbus & 3.4 & 1.1 & 1.0 & 14.1 & 11.7 & 10.0 & 4.7 & 1.3 & 1.0 \\
\hline 'Ballerina' & 2.0 & 1.0 & 1.0 & 19.1 & 13.2 & 11.4 & 2.9 & 1.0 & 1.0 \\
\hline Average value & 3.1 & 1.0 & 1.0 & 15.9 & 12.6 & 10.5 & 4.3 & 1.2 & 1.0 \\
\hline
\end{tabular}

BAP had a more significant impact on all morphometric indicators of shadberry explants in comparison with 2-IP. The number of new microshoots on the nutrient medium with BAP exceeded the number of microshoots on a 2-IP medium by 3 times, while the medium with 2-IP was not significantly different from the medium without a growth regulator. The smallest microshoots height in Amelanchier genus varieties was obtained on a nutrient medium without hormones addition and amounted to $10.5 \mathrm{~mm}$. The maximum height was achieved on a nutrient medium containing BAP amounting to $15.9 \mathrm{~mm}$. Explants cultivated on a nutrient medium containing 2-IP had an intermediate value of 12.5 $\mathrm{mm}$. The 2-IP growth regulator activated the growth of the lateral buds in 'Krasnoyarskaya' variety and the interspecies hybrid $\times$ Amelasorbus. On the nutrient medium with this hormone, explants of other genotypes became drawn in height, but did not form adventive microshoots. In turn, BAP induced the formation of advent shoots in the basal part of the explants. Due to the different action of growth regulators on shadberry explants, a significant difference in multiplication factors has also been revealed. On the nutrient medium without hormone addition, a minimum multiplication factor of 1.0 was noted. The 
multiplication factor for varieties on a medium containing BAP amounted to 4.3, on medium with 2-IP - 1.2.

A comparative analysis of shadberry varieties' regeneration capacity at different concentrations of 6-benzylaminopurin (Table 3) was also carried out.

Table 3. Influence of different BAP concentrations on morphometric indices of alder-leaf shadbush explants

\begin{tabular}{|c|c|c|c|c|}
\hline \multirow{3}{*}{ Variety } & $\begin{array}{c}\text { 6-BAP } \\
\text { concentration, } \\
\mathrm{mg} / \mathrm{L}\end{array}$ & $\begin{array}{c}\text { Height of } \\
\text { microshoots, } \\
\mathrm{mm}\end{array}$ & $\begin{array}{c}\text { Number of } \\
\text { microshoots, } \\
\text { pcs. }\end{array}$ & Multiplication factor \\
\hline \multirow{4}{*}{ 'Krasnoyarskaya' } & 0.2 & $19.0 \pm 1.4$ & $1.8 \pm 0.1$ & $1.8 \pm 0.1$ \\
\cline { 2 - 5 } & 0.3 & $17.4 \pm 1.2$ & $1.3 \pm 0.1$ & $1.9 \pm 0.2$ \\
\cline { 2 - 5 } & 0.5 & $14.8 \pm 1.4$ & $3.5 \pm 0.5$ & $5.9 \pm 1.0$ \\
\hline \multirow{4}{*}{ 'Mandan' } & 1.0 & $14.5 \pm 0.8$ & $4.8 \pm 0.6$ & $7.1 \pm 0.7$ \\
\cline { 2 - 5 } & 0.2 & $16.4 \pm 0.8$ & $1.5 \pm 0.1$ & $1.9 \pm 0.2$ \\
\cline { 2 - 5 } & 0.3 & $15.7 \pm 0.9$ & $1.9 \pm 0.1$ & $2.1 \pm 0.2$ \\
\cline { 2 - 5 } & 0.5 & $16.3 \pm 1.5$ & $3.0 \pm 0.4$ & $4.3 \pm 0.6$ \\
\hline \multirow{3}{*}{ 'Pmelasorbus } & 1.0 & $13.5 \pm 0.9$ & $3.4 \pm 0.2$ & $4.5 \pm 0.3$ \\
\cline { 2 - 5 } & 0.2 & $15.2 \pm 0.9$ & $1.2 \pm 0.1$ & $1.3 \pm 0.1$ \\
\cline { 2 - 5 } & 0.3 & $16.1 \pm 0.7$ & $1.3 \pm 0.1$ & $1.3 \pm 0.2$ \\
\cline { 2 - 5 } & 0.5 & $16.9 \pm 0.9$ & $1.8 \pm 0.2$ & $1.7 \pm 0.4$ \\
\hline & 1.0 & $14.1 \pm 2.2$ & $3.3 \pm 0.4$ & $3.0 \pm 0.6$ \\
\cline { 2 - 5 } & 0.2 & $12.0 \pm 1.2$ & $1.1 \pm 0.1$ & $1.7 \pm 0.1$ \\
\cline { 2 - 5 } & 0.3 & $11.9 \pm 0.9$ & $1.2 \pm 0.1$ & $1.7 \pm 0.1$ \\
\cline { 2 - 5 } & 0.5 & $13.9 \pm 1.3$ & $1.5 \pm 0.1$ & $2.7 \pm 0.2$ \\
\hline \multirow{3}{*}{ 'Ballerina' } & 1.0 & $18.8 \pm 0.7$ & $1.9 \pm 0.2$ & $4.7 \pm 0.2$ \\
\cline { 2 - 5 } & 0.2 & $11.7 \pm 0.6$ & $1.3 \pm 0.1$ & $1.5 \pm 0.1$ \\
\cline { 2 - 5 } & 0.3 & $13.6 \pm 1.1$ & $1.4 \pm 0.1$ & $1.6 \pm 0.1$ \\
\cline { 2 - 5 } & 0.5 & $15.1 \pm 1.1$ & $1.4 \pm 0.1$ & $1.6 \pm 0.1$ \\
\hline
\end{tabular}

A concentration of $0.5 \mathrm{mg} / \mathrm{L}$ 6-BAP was used as control. The results obtained showed that microshoots' height varied between 11.7 and $19.1 \mathrm{~mm}$. According to the microshoots' height, the studied genotypes were conditionally divided into three groups: microshoots' height increased with increasing growth regulator concentration ('Prince William' (A.canadensis), 'Ballerina' (A.×grandiflora)); microshoots' height decreased with increasing growth regulator concentration ('Krasnoyarskaya', 'Mandan' ( $A$. alnifolia)); microshoots' height did not change significantly at different growth regulator concentrations. In 'Prince William' and 'Ballerina' varieties, the microshoots (18.8 and 19.1 $\mathrm{mm}$ ) formed on the nutrient medium with $1.0 \mathrm{mg} / \mathrm{L}$ BAP exceeded the control option (13.9 and $15.1 \mathrm{~mm})$. The concentration of $0.2 \mathrm{mg} / \mathrm{L}$ contributed to the formation of low microshoots - 12.0 and $11.7 \mathrm{~mm}$. Microshoots' height on a nutrient medium containing $1.0 \mathrm{mg} / \mathrm{L}$ BAP in 'Krasnoyarskaya' variety - $14.5 \mathrm{~mm}$, and in the 'Mandan' variety - 13.5 $\mathrm{mm}$, which is significantly lower than the control option $(0.5 \mathrm{mg} / \mathrm{L} \mathrm{BAP})$. At a concentration of BAP equal to $0.2 \mathrm{mg} / \mathrm{L}$, the microshoots' height amounted to $19.0 \mathrm{~mm}$ in 'Krasnoyarskaya' variety and $16.4 \mathrm{~mm}$ in 'Mandan' variety.

The nutrient medium containing 6-benzylaminopurin at a concentration of $1.0 \mathrm{mg} / \mathrm{L}$ exceeded the control option and contributed to the formation of the largest microshoots' number; it was also characterized by a maximum multiplication factor in all shadberry varieties. The maximum microshoots number on medium with $1.0 \mathrm{mg} / \mathrm{L}$ BAP was noted in 'Krasnoyarskaya' variety $(4,8 \pm 0,6)$, as well as the multiplication factor $(7.1 \pm 0,7)$. The 
smallest microshoots number on this nutrient medium was shown by 'Prince William' $(1,9 \pm 0.2)$, and the minimum multiplication factor - 'Ballerina' (2.9 \pm 0.3$)$.

During the rhizogenesis features identification at the rooting stage depending on used auxin sources and their concentration, the significant influence of genotype and nutritional medium's hormonal composition on the number of alder-leaf shadbush varieties' roots formed from the explant was established (Table 4)

Table 4. Influence of growth regulators and genotype on rhizogenesis of alder-leaf shadbush varieties

\begin{tabular}{|c|c|c|c|}
\hline $\begin{array}{c}\text { Variety } \\
\left(\mathrm{LCD}_{05} 0.56\right)\end{array}$ & $\begin{array}{c}\text { Growth } \\
\text { regulators, } \\
\left(\mathrm{LCD}_{05} 1.33\right)\end{array}$ & $\begin{array}{l}\text { Number of } \\
\text { roots, pcs. }\end{array}$ & Rootage, $\%$ \\
\hline \multirow{6}{*}{ 'Krasnoyarskaya' } & $0.5 \mathrm{IAA}$ & 1.5 & 47 \\
\hline & $0.5 \mathrm{IBA}$ & 4.5 & 90 \\
\hline & $0.5 \mathrm{IAA}+0.5 \mathrm{IBA}$ & 2.3 & 29 \\
\hline & $1.0 \mathrm{IAA}$ & 1.8 & 38 \\
\hline & $1.0 \mathrm{IBA}$ & 4.7 & 90 \\
\hline & $1.0 \mathrm{IAA}+1.0 \mathrm{IBA}$ & 4.3 & 85 \\
\hline \multirow{6}{*}{ 'Mandan' } & $0.5 \mathrm{IAA}$ & 1.1 & 24 \\
\hline & $0.5 \mathrm{IBA}$ & 1.8 & 33 \\
\hline & $0.5 \mathrm{IAA}+0.5 \mathrm{IBA}$ & 1.3 & 67 \\
\hline & $1.0 \mathrm{IAA}$ & 2.0 & 29 \\
\hline & $1.0 \mathrm{IBA}$ & 2.6 & 71 \\
\hline & $1.0 \mathrm{IAA}+1.0 \mathrm{IBA}$ & 2.5 & 67 \\
\hline
\end{tabular}

The average number of roots formed in 'Krasnoyarskaya' variety is 3.8 pieces, which is significantly higher than 'Mandan' (2.0 pcs.). Nutrient media containing indolebutyric acid stimulated active growth of the root system. The maximum roots' number was obtained during cultivation with $1.0 \mathrm{mg} / \mathrm{L}$ IBA ('Krasnoyarskaya' - 4.7 pcs., 'Mandan' - 2.6 pcs.). The lowest roots' number was shown by a nutrient medium containing IAA at a concentration of $0.5 \mathrm{mg} / \mathrm{L}$ and resulted in $1.5 \mathrm{pcs}$ in 'Krasnoyarskaya' and 1.1 in 'Mandan' variety. It is worth noting that the largest percentage of rooted explants were also observed on a nutrient medium with $1.0 \mathrm{mg} / \mathrm{l}$ IBA ('Krasnoyarskaya' - 90\%, 'Mandan' - 71\%).

Shadberry depositing experiment was carried out on MS medium with $1 / 2$ mineral composition supplemented by $0.5 \mathrm{mg} / 1 \mathrm{BAP}$ and sucrose in various concentrations: 20.0 $\mathrm{g} / \mathrm{L}, 40.0 \mathrm{~g} / \mathrm{L}$ and $60.0 \mathrm{~g} / \mathrm{L}$. The temperature in the storage chamber was $15^{\circ} \mathrm{C}$, photoperiod - 16 h. After 12 months, the microshoots' height was measured, their number was calculated

The sucrose concentration did not affect the height and number of microshoots. The greatest height of all genotypes was shown by microshoots of 'Krasnoyarskaya' variety, the smallest - 'Thiessen'. The number of microshoots did not differ significantly in genotypes, it ranged from 1.5 to 2.5 pcs. After 12 months of depositing, shadberry explants remained viable.

\section{Conclusions}

Explants' regeneration features in varieties belonging to various species of Amelanchier genus have been identified. It was established that the Murashige-Skoog (1962) nutrient medium is optimal for shadberry varieties regeneration induction, and the cytokinin actiontype substance is 6-benzilaminopurin, which stimulates adventive microshoots' formation. The greatest morphogenetic potential among representatives of Amelanchier genus was shown by 'Krasnoyarskaya' A. alnifolia variety $(7,1)$. 
The varieties 'Prince William' (A. canadensis) and 'Ballerina' (A. ×grandiflora) showed an increase in microshoots' height depending on increase in growth regulators' concentration in the nutrient medium. The maximum microshoots' height was achieved in the experimental option with $1.0 \mathrm{mg} / \mathrm{L} 6$-BAP and amounted to 18.8 and $19.1 \mathrm{~mm}$ correspondingly. However, in genotypes belonging to $A$. alnifolia species ('Krasnoyarskaya', 'Mandan'), as well as in $\times$ Amelasorbus, reduction in the microshoots' height was observed with increased growth regulator concentration. The largest microshoots height was obtained on MS medium with $0.2 \mathrm{mg} / \mathrm{L}$ BAP $(19.0$ and $16.4 \mathrm{~mm}$ respectively).

The optimal growth regulator for A. alnifolia varieties at the rhizogenesis stage was IBA in of $1.0 \mathrm{mg} / \mathrm{L}$ concentration. Among this species' varieties, the maximum frequency of microshoots' root formation was noted in 'Krasnoyarskaya' variety (70\%).

Shadberry explants remain viable for 12 months when cultivated on the nutrient medium Murashige-Skoog (1962) with $1 / 2$ mineral composition containing $0.5 \mathrm{mg} / \mathrm{L}$ BAP and 20.0 to $60.0 \mathrm{~g} / \mathrm{L}$ sucrose.

\section{Acknowledgments}

The work was carried out within the framework of the State task of the SBS RAS (No.18118021490111-5)

\section{References}

1. T.E. Bocharova, N.V. Khromov, Agroecological aspects of sustainable AIC development, Materials of XV International Scientific Conference, 380-383 (2018)

2. L.G. Ermosh, N.V. Prisukhina, V.V. Kazina, Bulletin of KrasSAU, 12, 131-138 (2019)

3. T.N. Arkhipova, N.V. Krylova, Horticulture and Viticulture, 5, 12-13 (2006)

4. N.F. Teslenko, I.B. Krasina, O.A. Bogdanov, A.A. Fadeeva, Fundamental Research, 8, 333-337 (2015)

5. N.V. Chromov, Evaluation of shadberry gene pool by economic and biological traits and reproduction technology in the conditions of the Tambov region, Abstr. of the dis., 154 (2007)

6. A.V. Stepanova, Ecological and biological assessment of shadberry gene pool (Amelanchier Medik.) under introduction in the conditions of the CCR southwest, abstr. of the Dis., 174 (2015)

7. M.Kh. Tutov, Usage of raw materials of low-spread fruit breeds for the purpose of creating natural multivitamin drinks: abstract of the dis., 23 (2008)

8. V.L. Zakharov, O.A. Dubrovina, V.A. Gulidova, T.V. Zubkova, Bulletin of Michurinsk State Agrarian University, 3, 101-107 (2017)

9. S. Lachowicz, J. Oszmianski, S. Łukasz, P. Stanisła, Molecules, 22, 1-17 (2017)

10. I.F. Ovchinnikov, Shadberry, 32 (1963)

11. V.A. Vysotsky, Biotechnological methods in the system of revitated planting material production and breeding of fruit and berry plants, Abstr. of the dis., (Moscow), 44 (1998)

12. A.G. Kuklina, Honeysuckle, shadberry, 240 (2007)

13. K. Pruski, J. Nowak, G. Grainger, Plant Cell, Tissue and Organ Culture, 21,103-109 (1990) 
14. Y. Fengli, D. Baoguo, Indian Journal of Biotechnology, 16, 648-654 (2017)

15. M.H. Brand, Plant Cell, Tissue and Organ Culture 35, 203-209 (1993)

16. J. Hunková, G. Libiaková, J. Fejér, A. Gajdošová, Propagation of Ornamental Plants, 17(3), 103-107 (2017).

17. J. Hunkov, A. Gajdošová, Journal of Berry Research, 9, 549-561 (2019) 